converted by the operation into a pyo-pneumothorax. The improvement after the operation was only temporary, and in three weeks the patient was spitting up the same quantity of pus as before and was in much the same general condition, the temperature still not rising above the normal. It was then decided to lay the side freely open, which was done by extending the upper opening and passing a silver catheter through it to the sixth space posteriorly and cutting down upon its tip. The catheter carried a tape to which a drainage-tube was tied, and passed thus right through the pleural cavity from one opening to the other. A large amount of pus escaped. The chest was then covered with absorbent cotton-wool and picked oakum. The heart returned a little way towards the left after the operation. The expectoration diminished at once, and in the course of a week was hardly anything more than a little ordinary bronchitic sputum with an occasional small pellet of pus. The discharge from the wounds was at first copious, but rapidly decreased and remained throughout quite sweet, and the temperature never rose. The chest was syringed out twice daily with a 1 per cent. carbolic acid solution.

Ten days after the operation the expectoration had completely ceased, and the discharge from the incisions did not amount to more than three ounces in the twenty-four hours. Three days later the chest was filled rather too full of carbolic acid solution, and the patient coughed up a little of the acid, showing thus that the opening into the lung was still patent. One month after the operation the patient had gained four pounds in wejght; the discharge did not amount to more than an ounce in the twenty-four hours. In the fifth week the upper opening was allowed to close, and in the seventh the lower also, the patient having been up and about the wards for the last three weeks.

On discharge, two months and a half after the operation, the patient was strong, well, free from pain and expectoration, and had no dyspnoea over a moderate exertion. The left side of the chest was much flattened, and feeble respiratory sounds were audible over the upper lobe back and front, but none below. The heart had returned slightly towards the left, and the liver dulness had risen to the fifth rib on the right upper line.

The patient has been seen several times since, the last time nine months after the operation, and was at work and quite well.

Renuarks by Dr. WEST. - In this case an empyema had existed probably for nearly twelve months, and had been discharging externally and through the lung for about six months. The patient's health was good; there was no fever or hectic, and the nutrition was well maintained Under these circumstances the question was raised whether it would not be wisest to leave well alone; but considering the patient's discomfort, and the fact that if an operation were performed his chances of recovery were greatest while his strength and health were good, it was decided to run the necessary risks and lay the side freely open. The rapidity with which the expectoration ceased and the side contracted are surprising in an adult of his age and build. In performing the operation of making a counter-opening, there is, I think, no instrument so convenient as a stout wilver catheter-say a No. 10 . I have used it in many cases, and prefer it to any form of probe. This case is also an illustration of another fact of great importance. In empyemata, which discharge themselves, whether through the lung or externally, there is, as a rule, no pneumothorax. There is, of course, a communication between the pleura and the outer air-that is to say, there is a channel by which air might enter, but as a rule it does not do so. There was in this case no air in the pleura until some was sucked in by the aspirator, when the chest was tapped some days before the incision was made and this quickly dis appeared. In an ordinary case of pneumothorax air enters the pleura because the lung at once collapses; but when the lung is already collapsed, as it is in an empyema, and an opening either externally or into the lung is made, pus escapes until the positive pressure of the empyema is reduced to atmospheric pressure, and then ceases to flow but in most cases air does not enter the pleura when the opening is small, the pus merely, as it were, runs over when the pleura becomes too full. This appears to have been exactly the condition of things in the present case. The case was not treated antiseptically except that a 1 per cent. carbolic acid solution was used to wash the pleura out with, and that the wounds were covered with oakum and carbolised cottonwool.

\section{STROUD HOSPITAL.}

EPITHELIOMA OF PENIS ; AMPUTATION, WITH TRANSPLANTATION OF URETHRA THROUGH A HOLF IN THE PERINEUM.

(Under the care of Mr. STORRY.)

For the following notes we are indebted to Mr. Duncan R. Cameron, house-surgeon.

F. T_, a baker, aged fifty-two, was admitted into hospital on July 16th, 1883. He declared he had never had any chancre or clap. Nine months ago he noticed a small tumour about the size of a pea on the under part of the glans close to the frænum preputii. In a month's time the growth was as large as a hazel nut; and he began to suffer from the most painful priapisms. Up to four months ago he had been able always to uncover the glans penis.

The day following his admission Mr. Storry slit up the foreskin and removed a growth about the size of an ordinary hazel nut, together with a portion of the glans penis from the under side of the penis.

In a fortnight the patient was made an out-patient; but he presented himself in about six weeks' time with a return rowth and with some considerable infiltration of the penis. His great complaint was his terrible suffering at night from painful erections. There were no enlarged glands in the groin. Mr. Storry, under ether, amputated the penis, after the method adopted by Prof. Thiersch, a short account of which is given in the May number of the British Medical Journal for 1878. The urethra was dissected out and transplanted through a hole in the perineum, and a drainagetube was passed through the whole length of the scrotum. Within twenty-four hours the scrotum filled with blood, which caused the stitches to give way and, of course, delayed union. At the end of six weeks from the date of operation the patient was discharged with the wound almost healed, passing his urine easily and comfortably through the hole in the perineum, to the edges of which the urethra was attached. Three months after the operation he appeared at the hospital to show himself, having gained in flesh, and feeling "a new man," as he expressed himself, declaring he had no pain whatever, and could pass his urine with great ease and comfort, although he was obliged to take down his trousers altogether to micturate.

\section{SHEFFIELD PUBLTC HOSPITAL.}

FATAL CASE OF HYDROCHLORIC ACID POISONING; NECROPSY ; REMARKS.

(Under the care of Dr. DYSON.)

FOR the following notes we are indebted to Dr. Sinclair White.

Henry S_, aged fifty-four years, recently discharged from a lunatic asylum, was brought to the hospital on Nov. 12th, 1883. He stated that he had swallowed two tablespoonfuls of strong "spirits of salts" three hours previously.

On admission he was considerably collapsed; the skin was cold and clammy; the countenance anxious; the pulse small, weal, and frequent. He had vomited several times the vomited matter was dark in colour, viscid, contained blood, gave a strongly acid reaction with litmus, and a very copious white precipitate with a solution of nitrate of silver. On examining the mouth the mucous membrane was of a whitish colour, with here and there a red patch, showing where the epithelium had been removed. He complained of a burning sensation in the mouth, throat, and stomach ; and pressure over the latter viscus caused pain. He did not complain of thirst, even when interrogated. There was no diarrhoea or tenesmus. Solution of potash, freely diluted was administered, and afterwards lime-water and milk. Hot fomentations, sprinkled with laudanum, were applied over the stomach, and hot-water bottles to the feet. He passed a restless night, vomiting almost incessantly ; got weaker next morning, and died collapsed seventeen hours after swallowing the acid.

An examination of the body was made thirty-two hours after death. The mucous membrane of the cesophagus was highly congested in its upper half ; the lower half was black in colour, thickened and contracted; the veins stood out prominently, being filled with black blood. Just before its passage through the diaphragm there was a perforation in 